\title{
Amélioration continue de la qualité en chirurgie orthopédique: modifications et répercussions de la réforme du financement du système de santé
}

Fruit d'une collaboration spéciale, cet éditorial sur les mesures de contrôle de la qualité en chirurgie orthopédique arrive à point nommé: en effet, il est grand temps que des mesures d'amélioration de la qualité utiles et élaborées par des médecins soient adoptées. Nous ne voulons plus nous voir imposer des mesures élaborées et approuvées sans notre concours. Et pour que les choses changent, toutes les spécialités chirurgicales devront mettre la main à la pâte.

u Canada, les dépenses totales en santé prévues pour 2015 s'élevaient à 219,1 milliards de dollars, soit 6105 dollars par habitant ${ }^{1}$. Les soins orthopédiques comptaient pour environ $12 \%$ des dépenses associées aux soins actifs dans les hôpitaux². En 2012-2013, pour chaque dollar fiscal, 42 cents ont servi à financer les soins de santé en Ontario. Si rien ne change, les dépenses en santé pourraient représenter jusqu'à $70 \%$ du budget provincial d'ici $2025^{3}$.

C'est pour freiner cette augmentation que la réforme du financement du système de santé (RFSS) a été implantée en avril 2012 dans le cadre du Plan d'action de l'Ontario en matière de soins de santé. Parmi les principaux éléments de cette réforme, notons la mise en place d'un modèle normalizé de paiement regroupé pour les actes médicaux fondés sur la qualité (AMFQ) visant à encourager les soins qui améliorent les résultats pour les patients. D'autres pays, dont les États-Unis, tendent également vers l'adoption de modèles semblables ${ }^{4,5}$. Parmi les AMFQ actuellement posés en Ontario, 20 \% (c.-à-d. 4 sur 20) sont des interventions en chirurgie orthopédique ${ }^{6}$.

L'objectif derrière l'implantation de la RFSS et l'imposition d'AMFQ est le même : améliorer la qualité des soins de santé. C’est bien connu : en améliorant la qualité des soins et la satisfaction des patients, on obtient de meilleurs résultats pour les patients et une diminution des coûts. Mais en quoi consiste l'amélioration de la qualité? Quelle est la meilleure méthode pour la mettre en œuvre et l'évaluer?

\section{Quelle est la place de la qualité dans le contexte actuel des soins de santé?}

Selon l'Institute of Medicine ${ }^{7}$, des soins de qualité doivent être sécuritaires, efficaces, axés sur le patient, efficients, équitables et fournis dans des délais raisonnables. L'amélioration de la qualité ne passe pas nécessairement par une augmentation des dépenses : elle peut même servir à économiser de l'argent. En effet, une meilleure coordination des soins peut se traduire par une diminution du taux de complications, de la durée des séjours, des réadmissions et de l'utilization des services de santé après une chirurgie ${ }^{8}$. L'évaluation et l'amélioration de la qualité des soins reposent sur 3 dimensions séquentielles et interreliées, soit les structures, les processus et les résultats?.

De plus en plus, les données recueillies au moyen d'ensembles de données locaux sont transmises à des registres provinciaux et nationaux afin de favoriser les initiatives d'amélioration de la qualité ${ }^{10,11}$. Dans le cadre du National Surgical Quality Improvement Program de l'American College of Surgeons, on évalue et compare la qualité des soins chirurgicaux offerts en Amérique du Nord pour aider les hôpitaux à cibler certaines complications évitables ${ }^{12-16}$. Compte tenu de l'évolution constante des soins de santé attribuable aux diverses percées dans les technologies, les procédés et les connaissances médicales, il devient essentiel d'adopter un cycle de type « Planification-Exécution-ÉtudeAction », au moyen duquel il sera possible d'analyzer instantanément les données et de fournir constamment de la rétroaction aux fins de révision et d'amélioration continues ${ }^{16}$.

L'amélioration continue de la qualité (ACQ) repose directement sur ce type de cycle. Elle s'applique à tous les processus visant à fournir un produit ou un service à un consommateur de façon à combler ou à dépasser ses attentes. Toute initiative d'ACQ doit reposer sur une stratégie de planification cohérente servant à évaluer les structures et les processus actuels et à les améliorer pour atteindre la vision et les résultats souhaités ${ }^{17,18}$. Pour ce faire, il est de plus en plus fréquent dans le secteur des soins de santé, notamment en chirurgie orthopédique, d'avoir recours à la méthode des coûts par activités tout en tenant compte du temps requis ${ }^{19}$.

\section{ACQ et chirurgie orthopédique}

Parmi les principaux aspects de la plupart des programmes d'ACQ, notons : la collecte de données permettant l'évaluation des structures, des processus et des résultats pour les patients; le transfert aux chirurgiens et aux intervenants des données sur le rendement et les résultats, de préférence après qu'elles aient été ajustées en fonction du risque et étalonnées; la mise en œuvre d'interventions appropriées pour atténuer les variations inutiles entre les soins tout en améliorant le rendement ${ }^{20}$. 
Même si leur utilization dans le secteur des soins de la santé est relativement récente, les programmes d'ACQ ont fait leurs preuves pour améliorer les résultats pour des patients en chirurgie orthopédique, notamment dans le cadre d'une initiative dirigée par l'Alberta Bone and Joint Health Institute ${ }^{21,22}$. Dans certaines surspécialités de la chirurgie orthopédique, d'autres indicateurs de qualité ont également été recensés, comme la prévention des chutes ${ }^{23}$, l'administration d'antibiotiques au service des urgences ${ }^{24}$ et l'utilization immédiate de l'IRM en cas de lésions médullaires graves ${ }^{25}$.

En Ontario, les programmes d'amélioration de la qualité sont désormais obligatoires, et ils s'inscrivent dans le cadre des priorités du système et de la province ${ }^{24}$. Les chirurgiens doivent comprendre les principes fondamentaux de l'ACQ et la façon de les appliquer aux soins de leurs patients afin de jouer un rôle actif dans cette quête ${ }^{25}$. Deux facteurs sont essentiels à l'instauration d'une culture axée sur l'amélioration de la qualité et de la sécurité des patients dans le secteur des soins de santé : la collaboration et l'intégration. Il peut cependant être difficile d'améliorer concrètement le rendement pour différentes raisons touchant la qualité, la conception des processus de prestation des soins, l'évaluation des apports et des résultats, la collaboration des intervenants et les programmes d'incitation au rendement. Parmi les obstacles couramment signalés, notons le manque de temps, de ressources et de formation ainsi que les contraintes associées à d'autres changements ${ }^{26-29}$. Les futurs efforts d'amélioration de la qualité devront reposer sur un leadership affirmé des médecins afin que soit mise sur pied une équipe de soins optimale et axée le plus possible sur le patient ${ }^{8}$.

En résumé, les programmes d'ACQ visant des services de soins de santé peuvent orienter la prise de décisions et, grâce à l'application des connaissances, optimiser les soins et en améliorer l'efficacité. Parmi les résultats potentiels, soulignons une plus grande satisfaction des patients, une amélioration des résultats déclarés par les patients, des plans d'interventions plus efficaces et des économies globales.

\section{Paul E. Beaulé, MD; Darren M. Roffey, PhD; Stéphane Poitras, PHT, PhD}

Affiliations: Division de la chirurgie orthopédique, L'Hôpital d'Ottawa, Ottawa, Ont. (Beaulé); Programme d'épidémiologie clinique, Institut de recherche de L'Hôpital d'Ottawa, Ottawa, Ont. (Roffey); Programme de physiothérapie, Faculté des sciences de la santé, Université d'Ottawa, Ottawa, Ont. (Poitras).

Intérêts concurrents : Aucun déclaré.

DOI: $10.1503 /$ cjs.006316

\section{Références}

1. Canadian Institute for Health Information. National health expenditure trends, 1975 to 2015. 2015. Disponible : www.cihi.ca/sites /default/files/document/nhex_trends_narrative_report_2015_en.pdf (consulté le 11 mai 2016).
2. Waddell J. Ontario's experience with orthopaedic quality-based procedures and indicators. Advancing high-value practices for orthopaedic care. 11 May 2015. Toronto, Ont.

3. Ontario Ministry of Health and Long Term Care. Health system funding reform (HSFR). 2015. Disponible : www.health.gov.on.ca/en /pro/programs/ecfa/funding/hs_funding.aspx (consulté le 11 mai 2016).

4. Bozic KJ, Ward L, Vail TP, et al. Bundled payments in total joint arthroplasty: targeting opportunities for quality improvement and cost reduction. Clin Orthop Relat Res 2014;472:188-93.

5. Chernew ME. Why physicians should like bundled payment. Health Serv Res 2011;46:1693-7.

6. Ontario Ministry of Health and Long Term Care. Quality-based procedures. 2015. Disponible : www.health.gov.on.ca/en/pro/programs /ecfa/funding/hs_funding_qbp.aspx (consulté le 11 mai 2016).

7. Committee on Quality of Health Care in America. Crossing the quality chasm: a new bealth system for the 21st century. Institute of Medicine, Ed. Washington, DC: National Academy Press; 2001.

8. Saleh KJ, Bozic KJ, Graham DB, et al. Quality in orthopaedic surgeryan international perspective: AOA critical issues. 7 Bone foint Surg Am 2013;95:e3.

9. Donabedian A. Evaluating the quality of medical care. 1966. Milbank Q 2005;83:691-729.

10. Health Quality Ontario. What is quality improvement? 2016. Disponible : www.hqontario.ca/Quality-Improvement (consulté le 11 mai 2016).

11. Ontario Ministry of Health and Long Term Care. Quality improvement plans. 2016. Disponible : www.health.gov.on.ca/en/pro/programs /ecfa/legislation/quality_improve.aspx (consulté le 11 mai 2016).

12. Montroy J, Breau RH, Cnossen S, et al. Change in adverse events after enrollment in the National Surgical Quality Improvement Program: a systematic review and meta-analysis. PLoS One 2016;26:e146254.

13. Aoude AA, Aldebeyan SA, Nooh A, et al. Thirty-day complications of conventional and computer-assisted total knee and total hip arthroplasty: analysis of 103,855 patients in the American College of Surgeons National Surgical Quality Improvement Program database. 7 Arthroplasty 2016; doi: 10.1016.

14. Whiting PS, White-Dzuro GA, Avilucea FR, et al. Body mass index predicts perioperative complications following orthopaedic trauma surgery: an ACS-NSQIP analysis. Eur 7 Trauma Emerg Surg 2016; e-pub ahead of print.

15. Drager J, Hart A, Khalil JA, et al. Shorter hospital stay and lower 30-day readmission after unicondylar knee arthroplasty compared to total knee arthroplasty. 7 Artbroplasty 2016;31:356-61.

16. Su AW, Habermann EB, Thomsen KM, et al. Risk factors for 30-day unplanned readmission and major perioperative complications following spine fusion surgery in adults: a review of the National Surgical Quality Improvement Program (NSQIP) database. Spine 2016; e-pub ahead of print.

17. Goldstone $\mathrm{J}$. The role of quality assurance versus continuous quality improvement. 7 Vasc Surg 1998;28:378-80.

18. Health Information Technology Research Center (HITRC). Continuous quality improvement (CQI) strategies to optimize your practice 2013. Disponible : www.healthit.gov/sites/default/files/tools/nlc _continuousqualityimprovementprimer.pdf (consulté le 11 mai 2016).

19. Akhavan S, Ward L, Bozic KJ. Time-driven activity-based costing more accurately reflects costs in arthroplasty surgery. Clin Orthop Relat Res 2016;474:8-15.

20. Brindis RG, Dehmer GJ. Continuous quality improvement in the cardiac catheterization laboratory: Are the benefits worth the cost and effort? Circulation 2006;113:767-70.

21. Frank C, Marshall D, Faris P, et al. Joint Health I. Essay for the CIHR/CMAJ award: improving access to hip and knee replacement and its quality by adopting a new model of care in Alberta. CMAf 2011;183:E347-50.

22. Marshall DA, Christiansen T, Smith C, et al. Continuous quality improvement program for hip and knee replacement. Am 7 Med Qual 2015;30:425-31. 
23. Lohse GR, Leopold SS, Theiler S, et al. Systems-based safety intervention: reducing falls with injury and total falls on an orthopaedic ward. 7 Bone foint Surg Am 2012;94:1217-22.

24. Collinge CA, McWilliam-Ross K, Kelly KC, et al. Substantial improvement in prophylactic antibiotic administration for open fracture patients: results of a performance improvement program. 7 Orthop Trauma 2014:28:620-5.

25. Potigailo VL, Christoforidis GA, Katzman GL. Acute spinal cord compression: CQI framework increases resource efficiency while promoting delivery of high-quality care. 7 Am Coll Radiol 2015;12:90-4.

26. Ontario HQ. Quality improvement planning. 2016. Disponible : www.hqontario.ca/Quality-Improvement/Quality-Improvement-Plans (consulté le 11 mai 2016).
27. Bosco JA, Sachdev R, Shapiro LA, et al. Measuring quality in orthopaedic surgery: the use of metrics in quality management. Instr Course Lect 2014;63:473-85.

28. Baker GR, Barnsley J, Murray M. Continuous quality improvement in Canadian health care organizations. Leadersh Health Serv 1993;2:18-23.

29. Koenig KM, Bozic KJ. Orthopaedic healthcare worldwide: the role of standardization in improving outcomes. Clin Orthop Relat Res 2015;473:3360-3.

30. Wei DH, Hawker GA, Jevsevar DS, et al. Improving value in musculoskeletal care delivery: AOA critical issues. 7 Bone foint Surg Am 2015;97:769-74.

31. Hailer NP. Orthopedic registry research - limitations and future perspectives. Acta Orthop 2015;86:1-2.

\title{
ROYAL COLLEGE

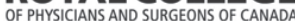 \\ COLLĖGE ROYAL
}

\section{Exclusive Royal College Grants for Surgeons}

\author{
Apply by Sept. 9, 2016
}

\section{Advance your surgical skills in the U.K. with the Harry S. Morton Travelling Fellowship in Surgery}

Up to $\$ 50,000 /$ year (maximum 2 years)

Surgeons and surgical residents in their final year of a Royal College accredited residency program can receive funding to train in the United Kingdom under the Royal College's Harry S. Morton Travelling Fellowship. This fellowship is a wonderful opportunity to benefit your practice and integrate excellence learned in the U.K. into Canada's academic programs.

\section{Host an invited speaker at your conference or event with the Harry S. Morton Lectureship in Surgery}

Up to $\$ 7,000 /$ lectureship for travel, accommodation and related expenses

Surgical National Specialty Societies and academic centres are eligible to apply for the Harry S. Morton Lectureship. These organizations may nominate a surgeon from the United Kingdom to travel to Canada to present at an academic conference or other surgical meeting. The lectureship encourages interactive discourse on emerging topics and innovative practices in a surgical specialty or subspecialty recognized by the Royal College.

Contact awards@ royalcollege.ca for more information. 